\title{
Optimal Vaccination Strategies of an SIR Epidemic Model with a Saturated Treatment
}

\author{
Hassan Laarabi*, Mostafa Rachik, Ouafa El Kahlaoui, El Houssine Labriji \\ Department of Mathematics and Computer Sciences, Faculty of Sciences Ben M'sik, \\ Bd Comandant Idriss El harti, 20450, Casablanca, Morocco \\ *Corresponding Author: hlaarabi@yahoo.fr
}

Copyright (C)2013 Horizon Research Publishing All rights reserved.

\begin{abstract}
In the present work, we consider a mathematical model of an SIR epidemic model with saturated incidence rate and saturated treatment function. We use an optimal vaccination strategies to minimize the susceptible and infected individuals and to maximize the number of recovered individuals. We work in the nonlinear optimal control framework. Some results concerning the existence and the characterization of the optimal control will be given. Numerical simulations are also presented.
\end{abstract}

Keywords Optimal control, Vaccination, SIR Models

\section{Introduction}

In recent years epidemiological modeling of infectious disease transmission has had an increasing influence on the theory and practice of disease management and control. Mathematical modeling of the spread of infectious diseases has become part of epidemiology policy decision making in many countries, (See for example: [2, 3, 4], $[6],[14],[15],[23],[26,27],[30])$. They can provide a powerful tool for investigating the dynamics and control of infectious diseases.

Optimal control theory provides a valuable tool to begin to assess the trade-offs between vaccination and treatment strategies [9], [11], [12], [13], [10], [18], [19], [20], [28]. Optimal control is a mathematical technique derived from the calculus of variations. Anyhow we can give suggestions to the public health authorities about the effects of a particular control policy with respect to others, and in this context the analysis and simulation of mathematical models may become a powerful tool in the hands of the above authorities. However, the control of epidemic systems is not usually an easy task since in real situations it is rather difficult to implement the control policies suggested by the mathematical analysis. There are a number of different methods for calculating the optimal control for a specific mathematical model. For example, Pontryagin's maximum principle [22] allows the calculation of the optimal control for an ordi- nary equation model system with a given constraint. The aim of this work is not to consider a special disease but to set up an optimal control problem relative to the SIR model. To do this, we use a percentage of susceptible populations as a control in our model. This percentage is a function of time. Hence, the optimal control (vaccination) strategy is to minimize the infected and susceptible individuals, and to maximize the total number of recovered individuals.

The paper is organized as follows: In Section 2, the model is described. In Section 3, we use Pontryagin's maximum principle to investigate analysis of control strategies and to determine the necessary conditions for the optimal control of the disease. Section 4 gives several numerical examples, and Section 5 concludes this work.

\section{2 mathematical model}

\subsection{Model Assumptions}

In this subsection, we first give the following basic assumptions

- The total population is divided into three subgroups: susceptible, infected and recovered individuals and S, I, R denote their numbers, respectively. Positive constants $\mathrm{A}, \delta, \mu, \epsilon$ are the recruitment rate of the population, the natural death rate of the population, the natural recovery rate of the infected individuals and the disease-related death rate, respectively.

- While contacting with infected individuals, the susceptible individuals become infected at a saturated incidence rate $\frac{\lambda S I}{1+k I}$, where $\lambda$ is positive and $\mathrm{k}$ is nonnegative.

- Through treatment, the infected individuals recover at a saturated treatment function $h(I)=\frac{r I}{1+\alpha I}$, where $\mathrm{r}$ is positive and $\alpha$ is nonnegative.

Wendi Wang (2006) introduced a staged treatment function which describes that the treatment rate is proportional to the number of the infective when the capacity of treatment is not reached, and otherwise, takes 
the maximal saturated level. This seems more reasonable than the usual linear function. Besides this, we know that the efficiency for treatment will be seriously affected if the infected individuals are delayed for treatment.

Zhang and Xianning (2008) added a parameter $\alpha$ in the treatment function to measure the extent of the effect of the infected being delayed for treatment. At the same time, in order to model the saturated phenomenon of the treatment mentioned above, we propose a function $h(I)$ with the form in the assumptions, where $\mathrm{r}$ is the cure rate (when $\alpha=0$, the saturated treatment function returns to the linear one).

In addition, the incidence rate will also come to a saturated situation as the infected individuals increase because of the psychological effect or the inhibition effect. Capasso and Serio introduced a saturated incidence rate $\frac{\lambda S(t) I(t)}{1+k I(t)}$ into the epidemic model which tends to a saturated level when I gets large, where $\lambda S(t) I(t)$ measures the infection force of the disease and $\frac{1}{(1+k I)}$ describes the psychological effect or inhibition effect from the behavioral change of the susceptible individuals when their number increases or from the crowding effect of the infected individuals. Although this incidence rate is more complex to deal with than the bilinear one, it seems more reasonable.

\subsection{The model}

The model to be studied takes the following form

$$
\left\{\begin{array}{l}
\dot{S}(t)=A-\delta S(t)-\frac{\lambda S(t) I(t)}{1+k I(t)}, \\
\dot{I}(t)=\frac{\lambda S(t) I(t)}{1+k I(t)}-(\delta+\epsilon+\mu) I(t)-\frac{r I(t)}{1+\alpha I(t)}, \\
\dot{R}(t)=\mu I(t)+\frac{r I(t)}{1+\alpha I(t)}-\delta R(t)
\end{array}\right.
$$

With initial condition for the above system: $S(0)=S_{0} \geq 0, I(0)=I_{0} \geq 0, R(0)=R_{0} \geq 0$

\section{The Optimal Vaccination}

We consider the control variable $u(t) \in U_{a d}$ to be the percentage of susceptible individuals being vaccinated per unit of time. Here $U_{a d}=\{u \mid u(t)$ is measurable, $0 \leq$ $\left.u(t) \leq u_{\max }<\infty, t \in\left[0, t_{\text {end }}\right]\right\}$ indicates an admissible control.

Now, we consider an optimal control problem to minimize the objective functional

$$
J(u)=\int_{0}^{t_{\text {end }}}\left[A_{1} S(t)+A_{2} I(t)+\frac{1}{2} \tau u^{2}(t)\right] d t
$$

subject to

$$
\left\{\begin{aligned}
\dot{S}(t) & =A-(\delta+u(t)) S(t)-\frac{\lambda S(t) I(t)}{1+k I(t)} \\
\dot{I}(t) & =\frac{\lambda S(t) I(t)}{1+k I(t)}-(\delta+\epsilon+\mu) I(t)-\frac{r I(t)}{1+\alpha I(t)} \\
\dot{R}(t) & =\mu I(t)+\frac{r I(t)}{1+\alpha I(t)}-\delta R(t)+u(t) S(t),
\end{aligned}\right.
$$

With $S(0)=S_{0} \geq 0, I(0)=I_{0} \geq 0, R(0)=R_{0} \geq 0$ here $A_{1}$ and $A_{2}$ are small positive constants to keep a balance in the size of $S(t)$ and $I(t)$, respectively. The square of the control variable reflects the severity of the side effects of the vaccination. In the objective functional, $\tau$ is a positive weight parameter which is associated with the control $u(t)$. The objective of our work is to minimize the infected and susceptible individuals and to maximize the total number of recovered individual by using possible minimal control variables $u(t)$.

\subsection{Existence of an Optimal Control}

For existence, we consider a control system (3) with initial conditions. Then, we rewrite (3) in the following form:

$$
\phi_{t}=B \phi+F(\phi)
$$

where

$$
\begin{gathered}
\phi=\left[\begin{array}{c}
S(t) \\
I(t) \\
R(t)
\end{array}\right] \\
B=\left[\begin{array}{ccc}
-(u(t)+\delta) & 0 & 0 \\
0 & -(\delta+\epsilon+\mu) & 0 \\
u(t) & \mu & -\delta
\end{array}\right] \\
F(\phi)=\left[\begin{array}{c}
A-\frac{\lambda S(t) I(t)}{1+k I(t)} \\
\frac{\lambda S(t) I(t)}{1+k I(t)}-\frac{r I(t)}{1+\alpha I(t)} \\
\frac{r I(t)}{1+\alpha I(t)}
\end{array}\right]
\end{gathered}
$$

and $\phi_{t}$ denote derivative of $\phi$ with respect to time $t$. Equation (4) is a non-linear system with a bounded coefficient. We set $D(\phi)=B \phi+F(\phi)$.

Now,

$$
\mathrm{F}\left(\phi_{1}\right)-F\left(\phi_{2}\right)=
$$

$\left[\begin{array}{c}A-\frac{\lambda S_{1}(t) I_{1}(t)}{1+k I_{1}(t)} \\ \frac{\lambda S_{1}(t) I_{1}(t)}{1+k I_{1}(t)}-\frac{r I_{1}(t)}{1+\alpha I_{1}(t)} \\ \frac{r I_{1}(t)}{1+\alpha I_{1}(t)}\end{array}\right]-\left[\begin{array}{c}A-\frac{\lambda S_{2}(t) I_{2}(t)}{1+k I_{2}(t)} \\ \frac{\lambda S_{2}(t) I_{2}(t)}{1+k I_{2}(t)}-\frac{r I_{2}(t)}{1+\alpha I_{2}(t)} \\ \frac{r I_{2}(t)}{1+\alpha I_{2}(t)}\end{array}\right]$

$$
=\left[\begin{array}{c}
-\lambda\left(\frac{S_{1}(t) I_{1}(t)}{1+k I_{1}(t)}-\frac{S_{2}(t) I_{2}(t)}{1+k I_{2}(t)}\right) \\
\lambda\left(\frac{S_{1}(t) I_{1}(t)}{1+k I_{1}(t)}-\frac{S_{2}(t) I_{2}(t)}{1+k I_{2}(t)}\right)-r\left(\frac{I_{1}(t)}{1+\alpha I_{1}(t)}-\frac{I_{2}(t)}{1+\alpha I_{2}(t)}\right) \\
r\left(\frac{I_{1}(t)}{1+\alpha I_{1}(t)}-\frac{I_{2}(t)}{1+\alpha I_{2}(t)}\right)
\end{array}\right]
$$


Therefore

$$
\begin{aligned}
\left|F\left(\phi_{1}\right)-F\left(\phi_{2}\right)\right| & =\left|-\lambda\left(\frac{S_{1} I_{1}}{1+k I_{1}}-\frac{S_{2} I_{2}}{1+k I_{2}}\right)\right|+\left|\lambda\left(\frac{S_{1} I_{1}}{1+k I_{1}}-\frac{S_{2} I_{2}}{1+k I_{2}}\right)-r\left(\frac{I_{1}}{1+\alpha I_{1}}-\frac{I_{2}}{1+\alpha I_{2}}\right)\right|+\left|r\left(\frac{I_{1}}{1+\alpha I_{1}}-\frac{I_{2}}{1+\alpha I_{2}}\right)\right| \\
& \leq 2 \lambda\left|\frac{S_{1} I_{1}}{1+k I_{1}}-\frac{S_{2} I_{2}}{1+k I_{2}}\right|+2 r\left|\frac{I_{1}}{1+\alpha I_{1}(t)}-\frac{I_{2}}{1+\alpha I_{2}}\right| \\
& \leq 2 \lambda\left|\frac{S_{1} I_{1}\left(1+k I_{2}\right)-S_{2} I_{2}\left(1+k I_{1}\right)}{\left(1+k I_{1}\right)\left(1+k I_{2}\right)}\right|+2 r\left|\frac{I_{1}\left(1+\alpha I_{2}\right)-I_{2}\left(1+\alpha I_{1}\right)}{\left(1+\alpha I_{1}\right)\left(1+\alpha I_{2}\right)}\right| \\
& \leq 2 \lambda\left|S_{1}(t) I_{1}+k S_{1} I_{1} I_{2}-S_{2} I_{2}-k S_{2} I_{1} I_{2}\right|+2 r\left|I_{1}-I_{2}\right| \\
& \leq 2 \lambda\left|S_{1} I_{1}-S_{2} I_{2}+k I_{1} I_{2}\left(S_{1}-S_{2}\right)\right|+2 r\left|I_{1}-I_{2}\right| \\
& \leq 2 \lambda\left|S_{1} I_{1}-S_{2} I_{1}+S_{2} I_{1}-S_{2} I_{2}+k I_{1} I_{2}\left(S_{1}-S_{2}\right)\right|+2 r\left|I_{1}-I_{2}\right| \\
& \leq 2 \lambda\left|I_{1}\left(S_{1}-S_{2}\right)+S_{2}\left(I_{1}-I_{2}\right)+k I_{1} I_{2}\left(S_{1}-S_{2}\right)\right|+2 r\left|I_{1}-I_{2}\right| \\
& \leq 2 \lambda\left(\left|I_{1}\right|\left|S_{1}-S_{2}\right|+\left|S_{2}\right|\left|I_{1}-I_{2}\right|+k\left|I_{1}\right|\left|I_{2}\right|\left|S_{1}-S_{2}\right|\right)+2 r\left|I_{1}-I_{2}\right| \\
& \leq\left|I_{1}-I_{2}\right|\left(2 \lambda\left|S_{2}\right|+2 r\right)+\left|S_{1}-S_{2}\right|\left(2 \lambda k\left|I_{1}\right|\left|I_{2}\right|+2 \lambda\left|I_{1}\right|\right) \\
& \leq\left(2 \lambda \frac{A}{\delta}+2 r\right)\left|I_{1}-I_{2}\right|+\left(2 \lambda k \frac{A^{2}}{\delta}+2 \lambda \frac{A}{\delta}\right)\left|S_{1}-S_{2}\right| \\
& \leq M\left(\left|S_{1}-S_{2}\right|+\left|I_{1}-I_{2}\right|\right) \\
& \quad w h e r e M_{1}=m a x\left(2\left(\frac{\lambda A}{\delta}+r\right) ; 2 \lambda \frac{A}{\delta}\left(\frac{k A}{\delta}+1\right)\right) \\
&
\end{aligned}
$$

Also, we get

$$
\left|D\left(\phi_{1}\right)-D\left(\phi_{2}\right)\right| \leq V\left|\phi_{1}-\phi_{2}\right|, \text { where } V=\max (M,\|B\|)<\infty .
$$

Thus, it follows that the function $\mathrm{D}$ is uniformly Lipschitz continuous. From the definition of the control $\mathrm{u}(\mathrm{t})$ and the restriction on $S(t), I(t)$ and $R(t) \geq 0$, we see that a solution of the system (4) exists (Birkhoff and Rota, 1989). Let us go back to the optimal control problem, (2) - (3). In order to find an optimal solution, first we find the Lagrangian and Hamiltonian for the optimal control problem (2) - (3). In fact, the Lagrangian of the optimal problem is given by

$$
L(S, I, u)=A_{1} S(t)+A_{2} I(t)+\frac{1}{2} \tau u^{2}(t) .
$$

We seek the minimal value of the Lagrangian. To accomplish this, we define the Hamiltonian $H$ for the control problem:

$$
H\left(S, I, R, u, \lambda_{1}, \lambda_{2}, \lambda_{3}, t\right)=L(S, I, u)+\lambda_{1}(t) \frac{d S(t)}{d t}+\lambda_{2}(t) \frac{d I(t)}{d t}+\lambda_{3}(t) \frac{d R(t)}{d t}
$$

where $\lambda_{1}(t), \lambda_{2}(t)$ and $\lambda_{3}(t)$ are the adjoint functions to be determined suitably.

Theorem 3.1 There exists an optimal control $u^{*}(t)$ such that

$$
J\left(u^{*}(t)\right)=\min _{u \in U_{a d}} J(u(t))
$$

subject to the control system (3) with initial conditions.

Proof. To prove the existence of an optimal control we use the result in (Lukes, 1982). Note that the control and the state variables are nonnegative values. In this minimizing problem, the necessary convexity of the objective functional in $u(t)$ is satisfied. The control space

$$
U_{a d}=\left\{u \mid u(t) \text { is measurable, } 0 \leq u(t) \leq u_{\max }<\infty, t \in\left[0, t_{\text {end }}\right]\right\}
$$

is also convex and closed by definition. The optimal system is bounded which determines the compactness needed for the existence of the optimal control. In addition, the integrand in the functional (2), $A_{1} S(t)+A_{2} I(t)+\frac{1}{2} \tau u^{2}(t)$ is convex on the control $u(t)$. Also, we can easily see that, there exist a constant $\rho>1$, positive numbers $\omega_{1}$ and $\omega_{2}$ such that $J(u(t)) \geq \omega_{2}+\omega_{1}\left(|u|^{2}\right)^{\frac{\rho}{2}}$. We conclude that there exists an optimal control. 


\subsection{Characterization of the optimal control}

In the previous section we showed the existence of an optimal control which minimize the functional (2) subject to system(3). In order to derive the necessary conditions for this optimal control, we apply Pontryagin's maximum principle to the Hamiltonian $H$.

Theorem 3.2 Let $S^{*}(t), I^{*}(t)$ and $R^{*}(t)$ be optimal state solutions with associated optimal control variable $u^{*}(t)$ for the optimal control problem (2) and (3). Then, there exist adjoint variables $\lambda_{1}, \lambda_{2}$ and $\lambda_{3}$ that satisfy

$$
\begin{aligned}
& \frac{d \lambda_{1}(t)}{d t}=-A_{1}+\lambda_{1}(t)\left(\delta+u(t)+\frac{\lambda I}{1+k I}\right)-\lambda_{2}(t)\left(\frac{\lambda I}{1+k I}\right)-\lambda_{3}(t) u(t) \\
& \frac{d \lambda_{2}(t)}{d t}=-A_{2}+\lambda_{1}(t)\left(\frac{\lambda S}{(1+k I)^{2}}\right)-\lambda_{2}(t)\left(\frac{\lambda S}{(1+k I)^{2}}-(\delta+\epsilon+\mu)-\frac{r}{(1+\alpha I)^{2}}\right)-\lambda_{3}(t)\left(\mu+\frac{r}{(1+\alpha I)^{2}}\right)(6) \\
& \frac{d \lambda_{3}(t)}{d t}=\lambda_{3}(t) \delta
\end{aligned}
$$

with transversality conditions

$$
\lambda_{i}\left(t_{\text {end }}\right)=0, i=1,2,3
$$

Furthermore, the optimal control $u^{*}(t)$ is given by

$$
u^{*}(t)=\max \left(\min \left(\frac{\left(\lambda_{1}(t)-\lambda_{3}(t)\right) S^{*}(t)}{\tau}, u_{\max }\right), 0\right)(8)
$$

Proof. We use the Hamiltonian (5) in order to determine the adjoint equations and the transversality conditions. From setting $S(t)=S^{*}(t), I(t)=I^{*}(t)$ and $R(t)=R^{*}(t)$, and differentiating the Hamiltonian with respect to $S$, $I$ and $R$, respectively, we obtain $(6)$.

And by using the optimality conditions we find

$$
\frac{\partial H}{\partial u}=\tau u^{*}(t)-\lambda_{1}(t) S^{*}+\lambda_{3}(t) S^{*}=0, \text { at } u=u^{*}(t)
$$

which gives

$$
u^{*}(t)=\frac{\left(\lambda_{1}(t)-\lambda_{3}(t)\right) S^{*}(t)}{\tau}
$$

Using the property of the control space, we obtain

$$
\left\{\begin{array}{lll}
u^{*}(t)=0 & \text { if } & \frac{\left(\lambda_{1}(t)-\lambda_{3}(t)\right) S^{*}(t)}{\tau} \leq 0 \\
u^{*}(t)=\frac{\left(\lambda_{1}(t)-\lambda_{3}(t)\right) S^{*}(t)}{\tau} & \text { if } & 0<\frac{\left(\lambda_{1}(t)-\lambda_{3}(t)\right) S^{*}(t)}{\tau}<u_{\max } \\
u^{*}(t)=u_{\max } & \text { if } \quad & \frac{\left(\lambda_{1}(t)-\lambda_{3}(t)\right) S^{*}(t)}{\tau} \geq u_{\max }
\end{array}\right.
$$

So the optimal control is characterized as

$$
u^{*}(t)=\max \left(\min \left(\frac{\left(\lambda_{1}(t)-\lambda_{3}(t)\right) S^{*}(t)}{\tau}, u_{\max }\right), 0\right)
$$

\subsection{The optimality system}

The optimal control and the state are found by solving the optimality system, which consists of the state system (3) with the initial conditions at $t=0$, the adjoint system (6) with the final conditions (7) and the characterization of the optimal control (8). 
So the optimality system is given by

$$
\left\{\begin{aligned}
\dot{S}(t)= & A-\left(\delta+\max \left(\min \left(\frac{\left(\lambda_{1}(t)-\lambda_{3}(t)\right) S^{*}(t)}{\tau}, u_{\max }\right), 0\right)\right) S(t)-\frac{\lambda S(t) I(t)}{1+k I(t)} \\
\dot{I}(t)= & \frac{\lambda S(t) I(t)}{1+k I(t)}-(\delta+\epsilon+\mu) I(t)-\frac{r I(t)}{1+\alpha I(t)}, \\
\dot{R}(t)= & \mu I(t)+\frac{r I(t)}{1+\alpha I(t)}-\delta R(t)+\max \left(\min \left(\frac{\left(\lambda_{1}(t)-\lambda_{3}(t)\right) S^{*}(t)}{\tau}, u_{\max }\right), 0\right) S(t) \\
\dot{\lambda_{1}}(t)= & -A_{1}+\lambda_{1}(t)\left(\delta+\max \left(\min \left(\frac{\left(\lambda_{1}(t)-\lambda_{3}(t)\right) S^{*}(t)}{\tau}, u_{\max }\right), 0\right)+\frac{\lambda I(t)}{(1+k I(t))}\right) \\
\dot{\lambda_{2}}(t)= & -A_{2}+\lambda_{1}(t)\left(\frac{\lambda S(t)}{(1+k I(t))^{2}}\right)-\lambda_{2}(t)\left(\frac{\lambda S(t)}{(1+k I)^{2}}-(\delta+\epsilon+\mu)-\frac{r}{(1+\alpha I(t))^{2}}\right)-\lambda_{3}(t)\left(\mu+\frac{r}{(1+\alpha I(t))^{2}}\right) \\
\dot{\lambda_{3}}(t)= & \lambda_{3}(t) \delta \quad\left(\frac{\lambda i n}{(1+k I(t))}\right)-\lambda_{3}(t) \max \left(\min \left(\frac{\left(\lambda_{1}(t)-\lambda_{3}(t)\right) S^{*}(t)}{\tau}, u_{\max }\right), 0\right) \\
& \text { with } \lambda_{1}\left(t_{\text {end }}\right)=0, \lambda_{2}\left(t_{\text {end }}\right)=0, \lambda_{3}\left(t_{\text {end }}\right)=0, S(0)=S_{0}, I(0)=I_{0}, R(0)=R_{0}
\end{aligned}\right.
$$

\section{Numerical Simulations}

\subsection{The improved GSS1 method}

The resolution of the optimality system (9) is created improving the Gauss Seidel - like implicit finite-difference method developed in [10] and denoted GSS1 method. It consists on discretizing the interval $\left[t_{0}, t_{\text {end }}\right]$ by the points $t_{k}=k l+t_{0}(k=0,1, \ldots, n)$, where $l$ is the time step. Next, we define the state and adjoint variables $S(t), I(t), R(t)$ $, \lambda_{1}(t), \lambda_{2}(t), \lambda_{3}(t)$ and the control $u(t)$ in terms of nodal points $S^{k}, I^{k}, R^{k}, \lambda_{1}^{k}, \lambda_{2}^{k}, \lambda_{3}^{k}$ and $u^{k}$ with $S^{0}, I^{0}, R^{0}$, $\lambda_{1}^{0}, \lambda_{2}^{0}, \lambda_{3}^{0}$ and $u^{0}$ as the state and adjoint variables and the controls at initial time $t_{0} \cdot S^{n}, I^{n}, R^{n}, \lambda_{1}^{n}, \lambda_{2}^{n}, \lambda_{3}^{n}$ and $u^{n}$ as the state and adjoint variables and the controls at final time $t_{\text {end }}$.

As it is well known, the approximation of the time derivative by its first-order forward-difference is given, for the first state variable $S(t)$, by $\frac{d S(t)}{d t}=\lim _{l \rightarrow 0} \frac{S(t+l)-S(t)}{l}$.

We use the scheme developed by Gumel et al. to adapt it to our case as following:

$$
\begin{aligned}
\frac{S^{k+1}-S^{k}}{l} & =A-\left(\delta+u^{k}\right) S^{k+1}-\frac{\lambda S^{k+1} I^{k}}{1+k I^{k}} \\
\frac{I^{k+1}-I^{k}}{l} & =\frac{\lambda S^{k+1} I^{k+1}}{1+k I^{k+1}}-(\delta+\epsilon+\mu) I^{k+1}-\frac{r I^{k+1}}{1+\alpha I^{k+1}} \\
\frac{R^{k+1}-R^{k}}{l} & =\mu I^{k+1}+\frac{r I^{k+1}}{1+\alpha I^{k+1}}-\delta R^{k+1}+u^{k} S^{k+1}
\end{aligned}
$$

By applying an analogous technology, we approximate the time derivative of the adjoint variables by their first-order backward-difference and we use the appropriated scheme as follows:

$$
\begin{aligned}
\frac{\lambda_{1}^{n-k}-\lambda_{1}^{n-k-1}}{l}= & -A_{1}+\lambda_{1}^{n-k-1}\left(\delta+u^{k}+\frac{\lambda I^{k+1}}{1+k I^{k+1}}\right)-\lambda_{2}^{n-k}\left(\frac{\lambda I^{k+1}}{1+k I^{k+1}}\right)-\lambda_{3}^{n-k} u^{k} \\
\frac{\lambda_{2}^{n-k}-\lambda_{2}^{n-k-1}}{l}= & -A_{2}+\lambda_{1}^{n-k-1}\left(\frac{\lambda S^{k+1}}{\left(1+k I^{k+1}\right)^{2}}\right)-\lambda_{2}^{n-k-1}\left(\frac{\lambda S^{k+1}}{\left(1+k I^{k+1}\right)^{2}}-(\delta+\epsilon+\mu)\right. \\
& \left.-\frac{r}{\left(1+\alpha I^{k+1}\right)^{2}}\right)-\lambda_{3}^{n-k}\left(\mu+\frac{r}{\left(1+\alpha I^{k+1}\right)^{2}}\right) \\
\frac{\lambda_{3}^{n-k}-\lambda_{3}^{n-k-1}}{l}= & \lambda_{3}^{n-k-1} \delta
\end{aligned}
$$

\section{$4.2 \quad$ Numerical results}

We use the following data:

$S_{0}=1000 ; I_{0}=110 ; R_{0}=61 ; \mu=0.1 ; A=150 ; \alpha=0.01 ; \delta=0.1 ; \tau=10^{5} ; r=0.8 ; k=0.1 ; A_{1}=100 ; A_{2}=10 ; \gamma=$ $0.003 ;=0.1 ; \epsilon=0.01$;

We obtain the following figures (see Figure1)

We can see that the optimal vaccination and treatment function have a very desirable effect upon the population of infectious and susceptible which decreases while the recovered population increases for almost the entire length of therapy. 

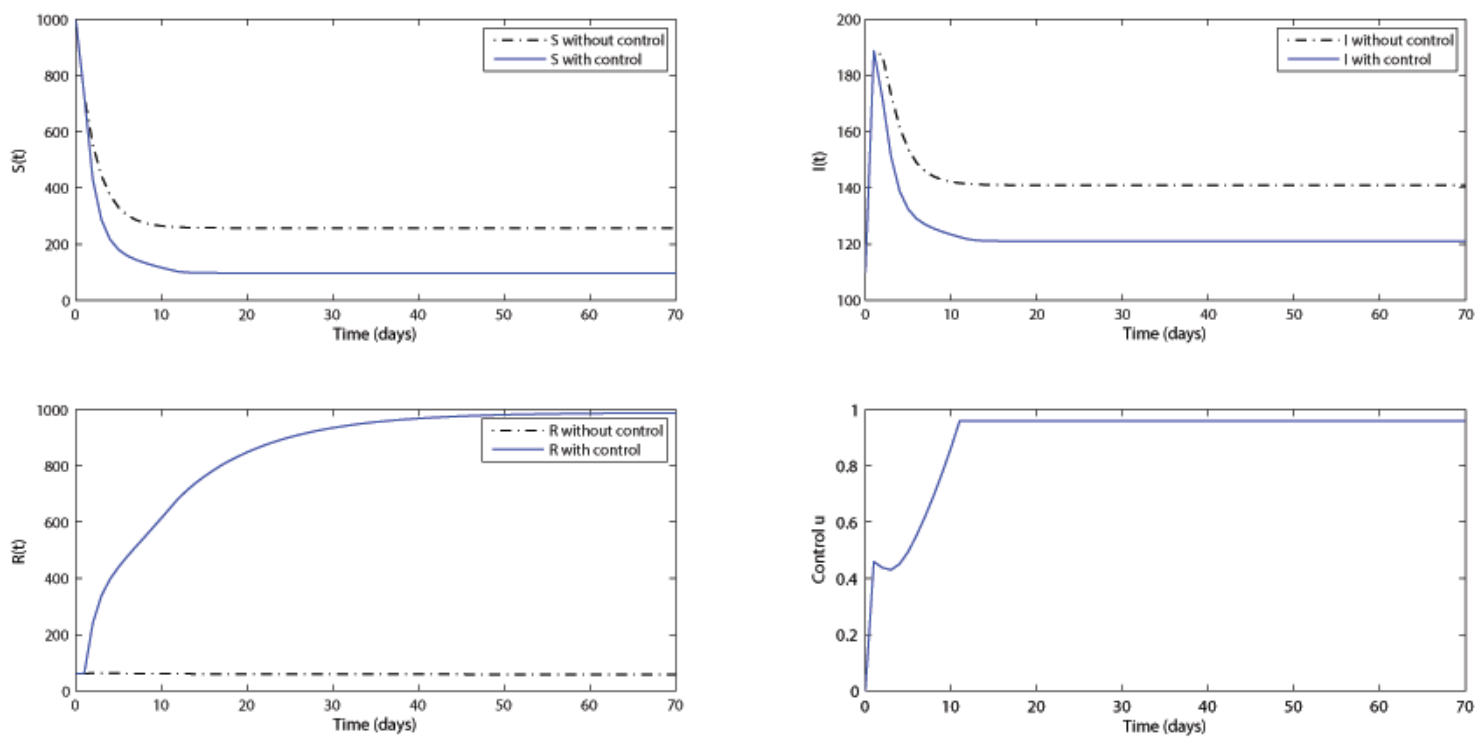

Figure 1. The plot shows the population both with control and no control.

\section{Conclusion}

In this paper we do not consider any special disease but our aim is to set up an optimal control problem relative to epidemic model with saturated incidence rate and saturated treatment function, so it is to minimize the infected and susceptible populations and to maximize recovered populations.

A comparison between individuals with optimal control and no control is presented. It is easy to see that the optimal control is much more effective for reducing the number of infected individuals. In order to illustrate the overall picture of the epidemic, the numbers of infected, susceptible and recovered individuals under the optimal control and no control are shown in figures.

So, we can see that the population of recovered individuals with control is more sharply increased than the individuals without control. The population of susceptible and infected with control is more sharply decreased than the population without control.

\section{REFERENCES}

[1] Birkhoff G., Rota G.C., Ordinary Differential Equations, 4th ed. John Wiley \& Sons, New York, (1989).

[2] F. Brauer, The Kermack-McKendrick epidemic model revisited. Math. Biosci. 198, pp. 119-131, (2005).

[3] F. Brauer, Some simple epidemic models. Math. Biosci. Eng. 3, pp. 1-15, (2006).

[4] F. Brauer, C. Castillo-Chavez, Mathematical models in population biology and epidemiology. In: Texts in Applied Mathematics, vol. 40. Springer, New York, (2001).

[5] V. Capasso, G. Serio, A generalization of the Kermack- Mckendrick deterministic epidemic model, Math. Biosci. 42, pp. 41-61, (1978).

[6] O. Diekmann, J.A.P Heesterbeek, Mathematical epidemiology of infectious diseases. Model building, analysis and interpretation. In: Wiley Series in Mathematical and Computational Biology. John Wiley \& Sons, Chichester, (2000).

[7] D.L. Lukes, Differential Equations: Classical to Controlled, Math. Sci. Eng. 162, Academic Press, New York, (1982).

[8] D. Xiao, S. Ruan, Global analysis of an epidemic model with nonmonotone incidence rate, Math. Biosci. 208, pp. 419-429, (2007).

[9] C.P. Farrington, On vaccine efficacy and reproduction numbers. Math. Biosci. 185, pp. 89-109, (2003).

[10] A.B. Gumel, P.N. Shivakumar, B.M. Sahai, A qualitative study of a vaccination model with nonlinear incidence. Appl. Math. Comput. 143, pp. 409-419, (2003).

[11] K. Hattaf, A. Lashari, Y. Louartassi and N. Yousfi, Two optimal treatments of HIV infection model, pp. 1-9, (2013).

[12] K. Hattaf and N. Yousfi, Optimal Control of a Delayed HIV Infection Model with Immune Response Using an Efficient Numerical Method, ISRN Biomathematics, Vol. 2012 (2012), Article ID 215124, 7 pages. 
[13] K. Hattaf and N. Yousfi, Two optimal treatments of HIV infection model, World Journal of Modelling and Simulation, 8 pp.27-35, (2012).

[14] H.W. Hethcote, The mathematics of infectious diseases, SIAM Rev. 42 pp. 599-653, (2000).

[15] Hsu S., Zee A, Global spread of infectious diseases, J. Biol. Syst. 12, pp. 289-300, (2004).

[16] J. Karrakchou, M. Rachik, S. Gourari, Optimal control and infectiology: Application to an HIV/AIDS model, Applied Mathematics and Computation 177, pp. 807-818, (2006).

[17] H.R. Joshi, Optimal control of an HIV immunology model, Optim. Control Appl. Methods 23, pp. 199-213, (2002).

[18] C.M. Kribs-Zaleta, J.X. Velasco-Hernandez, A simple vaccination model with multiple endemic states. Math. Biosci. 164, pp. 183-201, (2000).

[19] H. Laarabi, A. Abta, M. Rachik and J. Bouyaghroumni, Optimal Antiviral Treatment Strategies of HBV Infection Model with Logistic Hepatocyte Growth, ISRN Biomathematics, Vol. 2013 (2013), Article ID 912835, 6 pages.

[20] H. Laarabi, E. Labriji, M. Rachik, and A. Kaddar, Optimal control of an epidemic model with a saturated incidence rate, Nonlinear Analysis: Modelling and Control, vol. 17, no. 4, pp. 448-459, (2012).

[21] S. Lenhart, J.T. Workman, Optimal Control Applied to Biological Models. Mathematical and Computational Biology Series, Chapman \& Hall/CRC, London, (2007).

[22] L.S. Pontryagin, V.G. Boltyanskii, R.V. Gamkrelize, E.F. Mishchenko, The Mathematical Theory of Optimal Processes, New York, Wiley \& Sons, (1962).

[23] S.G. Ruan, W.D. Wang, Dynamical behaviour of an epidemic model with nonlinear incidence rate. J. Differ. Equations 188, pp. 135-163, (2003).

[24] Shulgin B, Stone L, Agur Z., Pulse vaccination strategy in the SIR epidemic model. Bull. Math. Biol. 60, pp. 1123-1148, (1998).

[25] W.H. Fleming, R.W. Rishel, Deterministic and Stochastic Optimal Control, Springer, New York. (1975).

[26] W. Wang, Backward bifurcation of an epidemic model with treatment, Math. Biosci., 201, pp. 58-71, (2006).

[27] W. Wang, Z. Ma, Global dynamics of an epidemic model with time delay. Nonlinear Anal. Real World Appl. 3, 365, (2002).

[28] G. Zaman, Y. Kang, H. Jung, Stability analysis and optimal vaccination of an SIR epidemic model, BioSystems 93, pp. 240-249, (2008).

[29] X. Zhang, X. Liu, Backward bifurcation of an epidemic model with saturated treatment, Journal of Mathematical Analysis and Applications, 348, pp. 433-443, (2008).

[30] Zhang H., Jiao J., Chen L, Pest management through continuous and impulsive control strategies. Biosystems 90, pp. 350-361, (2007). 